\title{
Analysis of Fresnel Loss at Splice Joint Between Single-Mode Fiber and Photonic Crystal Fiber
}

\author{
Samira Farsinezhad ${ }^{1}$, Faramarz E. Seraji ${ }^{2, *}$ \\ ${ }^{1}$ Excitonics and Nanostructures Laboratory, University of Alberta, Canada \\ ${ }^{2}$ Optical Communication Group, Iran Telecom Research Center, Tehran, Iran
}

\begin{abstract}
An analysis of Fresnel loss at splice joint of single-mode fiber (SMF) and two types of photonic crystal fibers (PCFs) using improved full-vectorial effective index methods is presented. The effects of air-hole size $(d)$, air-hole spacing $(\Lambda)$, longitudinal misalignment between spliced SMFs and PCFs on the Fresnel loss is analyzed and is shown that when $\Lambda$ and the dopant concentration in the core of PCFs increases or the ratio $d / \Lambda$ decreases, the Fresnel loss will decrease. The results of the analysis may be used by network designers to predict over all loss when employing PCF-based devices in optical fiber networks.
\end{abstract}

Keywords Fresnel loss, Single-mode fiber, Photonic crystal fiber

\section{Introduction}

In recent years, due to growth of optical communication networks, the splice joint between single-mode fibers (SMFs) as a transmission medium, and photonic crystal fiber (PCF) based devices has attracted more research work. One of the issues at the splice joint of SMFs and PCF-based devices worthy to attend is the reflection of light ray called as Fresnel reflection which is due to a possible change of refractive index at the splice point[1]. This phenomenon causes development of extra loss at the joint.

In the early research works, losses due to Fresnel reflection between SMFs were well reported[2-4]. The recent reports focused on the loss at the splice joints between SMF and PCF, where the attempts were made to optimize the spliced PCF parameters such as air-hole spacing $\Lambda$ and air-filling factor $d / \Lambda$ for reduction of the loss resulting from coupling mechanism between them[5-8].

In the reported investigations[5,8], the core refractive index of the spliced fibers was assumed to be equal and there was no indication of the influence of effective refractive index of PCFs on the loss due to Fresnel reflection. By creating a critical angle of 8 degrees at the end faces of spliced SMFs and hollow core PCFs, the Fresnel reflection may be avoided [1].

In connecting the PCF-based devices to SMFs, we should consider the influences of $\Lambda$ and $d / \Lambda$ on the Fresnel loss at the joint [9]. The presence of air-holes in the cladding region of the PCFs causes change in behavior of the

* Corresponding author:

feseraji@itrc.ac.ir (Faramarz E. Seraji)

Published online at http://journal.sapub.org/optics

Copyright (C) 2012 Scientific \& Academic Publishing. All Rights Reserved joint which can be studied using effective refractive indices of the spliced fibers[5,6]. In our previous reports, a mechanism was proposed using improved fully vectorial effective index method (IVEIM) to optimize splice joint of PCFs and SMFs[10]. In this paper, for the first time to our knowledge, we report an analysis of Fresnel loss at the splice joint of SMF and solid core PCFs.

In the analysis of the present paper, by using IVEIM[11, 12], we will consider the splice joints between single-mode fiber and two structurally different PCFs, i.e., conventional PCF (CPCF) and raised-core PCF (RCPCF)[13] to investigate the influences of PCFs parameters $\Lambda$ and $d / \Lambda$ on reduction of Fresnel loss at the splice joint.

\section{Formulation of Fresnel loss for Splice joint}

To calculate the Fresnel loss, Fig. 1 is considered for a splice of the fibers where their end facets, at a distance $D$ apart, are assumed parallel to each other. The incident rays are assumed perpendicular to the end facets. The ray transmission from fiber 1 to fiber 2 experiences reflections twice, one at the interface between end facet of fiber 1 and the air gap, and the other between the air gap and the entering facet of fiber 2 .

In general, in Fig. 1(a), the two co-directional rays (one of them is shown), normal to the plane wave front in fiber 1 after crossing medium $n_{2}$, strike the front end of fiber 2 and then are transmitted partially into medium $n_{3}$. For the net amplitude reflection coefficient $r$, the Fresnel coefficients for each boundary are used with a phase difference of $\beta$. We note that the Fresnel coefficients are different for $s$-and $p$ -polarization.

Therefore, we can write the following expressions for $r$ 
[14]:

$$
r=\frac{r_{12}+r_{23} e^{i \beta}}{1-r_{23} r_{21} e^{i \beta}}
$$

With similar derivation, we can write the net amplitude transmitted into fiber 2 as:

$$
t=\frac{t_{12} t_{23} e^{0.5 i \beta}}{1+\mathrm{r}_{12} \mathrm{r}_{23} \mathrm{e}^{i \beta}}
$$

where $r_{n m}$ is the intensity reflection coefficient from medium $(n)$ to medium $(m), t_{\mathrm{nm}}$ is the respective intensity transmission coefficient, and $\beta=2 \mathrm{D}(\omega / \mathrm{c})(\mathrm{p}+\mathrm{iq})$ is the phase difference, where $p=n_{2} \cos \left(\theta_{2}\right)$ and $q=0$ in a nonconducting medium for all angles of incidence, $\omega$ is the angular frequency of light, and $c$ is the velocity of light in a vacuum.

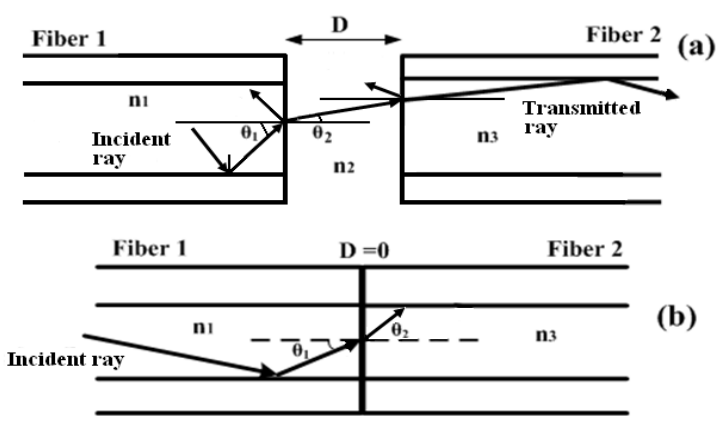

Figure 1. Demonstration of Fresnel loss at the splice joint of any two fibers (a) $D \neq 0$ and (b). $D=0$

In Eqs (1) and (20) the identities $r_{12}=-r_{21}, r_{12}^{2}+t_{12} t_{21}=1$ are used. So based on Fresnel coefficient, we can write the intensity reflectance $R$ as:

$$
R=\frac{\left(r_{12}^{2}+r_{23}^{2}+2 r_{12} r_{23} \cos \beta\right)}{\left(1+r_{12}^{2} r_{23}^{2}+2 r_{12} r_{23} \cos \beta\right)}
$$

For nonconducting media 1 and $3(q=0)$, and for real $\beta$ and real Fresnel coefficients we can write intensity reflectance and intensity transmittance as:

$$
\begin{aligned}
& R=\frac{R_{1}+R_{2}+2 \sqrt{R_{1} R_{2}} \cos \beta}{1+R_{1} R_{2}+2 \sqrt{R_{1} R_{2}} \cos \beta} \\
& T=\frac{1+R_{1} R_{2}-R_{1}-R_{2}}{1+R_{1} R_{2}+2 \sqrt{R_{1} R_{2}} \cos \beta}
\end{aligned}
$$

where $R_{1}=r_{12}^{2}$ and $R_{2}=r_{23}^{2}$ indicate the reflectance between fiber 1 and the air-gap, and between the air-gap and fiber 2, respectively[14].

With reference to Fig. 1(a), at normal incidence for $D \neq 0$, the reflectance for both $s$ - and $p$-polarization and the phase difference $\beta$ can be shown as[15]:

$$
r_{12}^{2}=\frac{\left(n_{1}-n_{2}\right)^{2}}{\left(n_{1}+n_{2}\right)^{2}}, r_{23}^{2}=\frac{\left(n_{2}-n_{3}\right)^{2}}{\left(n_{2}+n_{3}\right)^{2}}, \quad \beta=2 \mathrm{Dn}_{2} \frac{\omega}{c}
$$

The corresponding Fresnel loss will be:

$$
\text { Loss }\left._{\text {Fresnel }}\right|_{D \neq 0}=-10 \log (1-R)
$$

where all the parameters are indicated in Fig. (1). The corresponding transmittance for $D=0$ is obtained as:

$$
T=\frac{I_{\mathrm{t}}}{I_{\text {in }}}=\frac{n_{3}}{n_{1}} t_{12}^{2}=\frac{4\left(n_{1} n_{3}\right)}{\left(n_{1}+n_{3}\right)^{2}}
$$

where $I_{\mathrm{t}}$ and $I_{\text {in }}$ are the transmitted and incident intensities, respectively. Then the loss due to the Fresnel reflections can be derived as[2, 15]:

$$
\text { Loss Fresnel }_{D=0}=-10 \log \left[\frac{4 n_{1} n_{3}}{\left(n_{1}+n_{3}\right)^{2}}\right]
$$

Eq. (4) shows that the reflection and the transmission are implicitly dependent on three refractive indices $n_{1}, n_{2}$ and $n_{3}$, as shown in Fig.1. In addition, to eliminate the Fresnel loss at the splice joint, we should set $T=1$ (or $R=0$ ). With this precondition, we obtain an expression for maximum transmission at the splice joint as: $n_{2}=\left(n_{1} n_{3}\right)^{1 / 2}$. Since for $D \neq 0, n_{2}$ can be the refractive index of the air-gap $\left(n_{2} \approx 1\right)$, the condition for zero Fresnel loss will be $n_{1} n_{3} \approx 1$.

With the above discussion, let us now consider the splice between an SMF and a PCF. To be more specific on the light propagation through fibers at splice joint, we can presume that the light sees an effective refractive index rather mere core refractive index, which agrees usually with a practical condition. Under this situation, the influences of PCF parameters $\Lambda$ and $d / \Lambda$ can bring us the structural effects on the Fresnel loss at the splice point of the SMF and the PCF.

Now, to start with our study, we assume a perfect splice between the SMF (fiber 1) and the PCF (fiber 2), i.e., a splice with no longitudinal displacement $(D=0)$. Therefore, Eq. (8) reduces to:

$$
\left.\operatorname{Loss}_{F r e s n e l}\right|_{D=0}=-10 \log \left[\frac{4 n_{e f f}^{S M F} n_{e f f}^{P C F}}{\left(n_{e f f}^{S M F}+n_{e f f}^{P C F}\right)^{2}}\right]
$$

where $n_{\text {eff }}^{\mathrm{SMF}}$ and $n_{\text {eff }}^{\mathrm{PCF}}$ are the effective refractive indices of the SMF and the PCF, respectively.

\section{Numerical Results}

We consider splicing of SMF to a CPCF and a RCPCF in a separate calculation. To determine optimal values of refractive indices of silica cores of the spliced fibers, $n_{\mathrm{eff}}^{\mathrm{SMF}}$ of the SMF, $n_{\text {eff }}^{\text {CPCF }}$ of the CPCF, and $n_{\text {eff }}^{\text {RCPCF }}$ of the RCPCF, we used Sellemier equation and numerical IVEIM method, respectively, which are generally utilized[10, 11, 16,17].

The numerical results for $n_{\text {eff }}^{\text {CPCF }}$ and $n_{\text {eff }}^{\text {RCPF }}$ are illustrated in Fig. 2 in terms of $d / \Lambda$ for different values of $\Lambda$. We note that the effective refractive index as a function of $\Lambda$, experiences higher variations as $d / \Lambda$ increases. The range of this variation is less in case of the RCPCF.

For the splice joints of the SMF to the CPCF and the RCPCF [10, 13], the Fresnel losses, based on Eq. (9), in terms of $d / \Lambda$ for different values of $\Lambda$ are illustrated in Fig. 3 using IVEIM method. When $\Lambda$ increases, the Fresnel loss will decrease whereas increase of $d / \Lambda$ would cause an increase in the Fresnel loss for a given $\Lambda$, as shown in Fig. 3.

For higher values of $\Lambda$, the slopes of the curves decrease, showing a lesser influence of the ratio $d / \Lambda$. It is noted that with the same values of $\Lambda$ in Fig. 3(a), the replacement of CPCF with RCPCF in a splice with SMF has caused reduc- 
tions of the Fresnel loss. As $\Lambda$ increases, this effect is more prominent, as indicated in Fig. 3(b). In fact, at higher values of $\Lambda$, the Fresnel loss changes linearly and its dependency on $d / \Lambda$ will be weak. For instance, when $\Lambda=6.30 \mu \mathrm{m}$ and $d / \Lambda=0.345$, we observe more than $50 \%$ reduction of the Fresnel loss when using RCPCF[18].
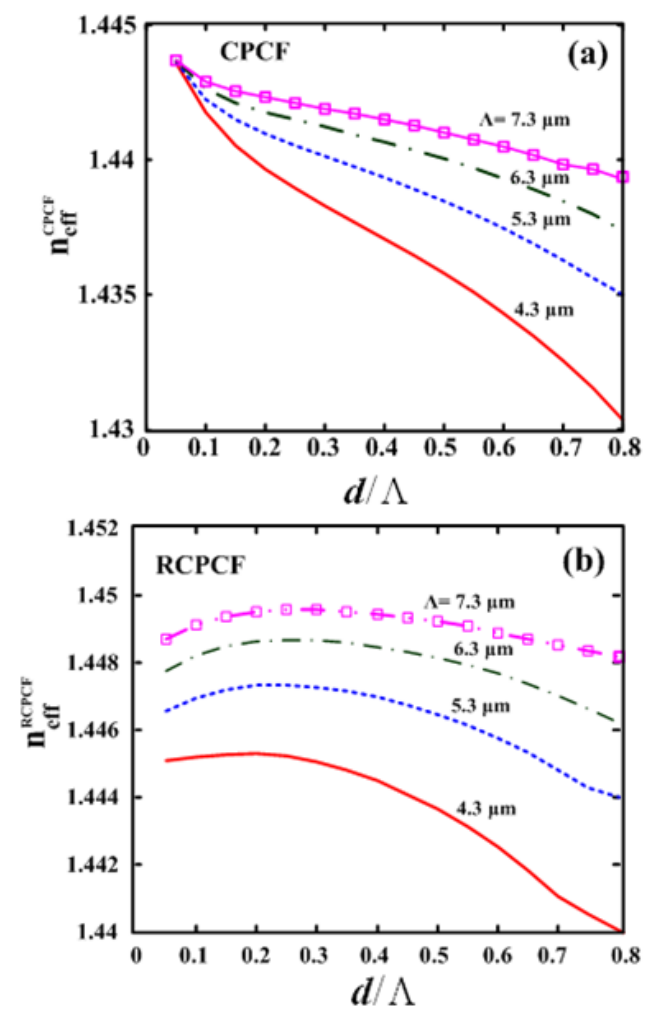

Figure 2. Calculations of the effective refractive index (a) $n_{\text {eff }}^{\text {CPCF }}$ and (b) $n_{\text {eff }}^{\text {RCPCF }}$ by using IVEIM at $1.55 \mu \mathrm{m}$.

Now, if there is a longitudinal displacement $(D \neq 0)$ at the splice joint between two fibers, the Fresnel loss will be determined from Eq. (6). Figure 4 illustrates the Fresnel loss for two cases: $d / \Lambda=0.2$ and $d / \Lambda=0.5$ for different values of $\Lambda$ at $1.55 \mu \mathrm{m}$. For all values of $\Lambda$ and $d / \Lambda$, all the minima at $D=0.39,1.16,1.94 \mu \mathrm{m}$ remain almost constant at low level $0.001 \mathrm{~dB}$, as shown in Fig. 4(a). The maxima of the Fresnel losses change slightly for higher values of $\Lambda$. When $d$ increases, the maximum Fresnel loss will go higher, as shown in Figs. 4(b) and 4(c). If $d / \Lambda$ is assumed constant, the Fresnel loss maintains sinusoidal changes with almost zero values at some longitudinal displacements points that do not alter with parameter changes, as these points depend on the ratio $D / \lambda$.

In fact, for lower values of $\Lambda$, when there is longitudinal displacement at the splice joint, the Fresnel loss dose not depend on structural parameters of the PCFs. It is reminded the presence of longitudinal displacement at the splice joints of two fibers causes two reflections.

One of the approaches to nullify the Fresnel loss at the splice joint is to maintain the following condition:

$$
n_{2}=\left(n_{\mathrm{eff}}^{\mathrm{SMF}} n_{\mathrm{eff}}^{\mathrm{PCF}}\right)^{1 / 2}
$$
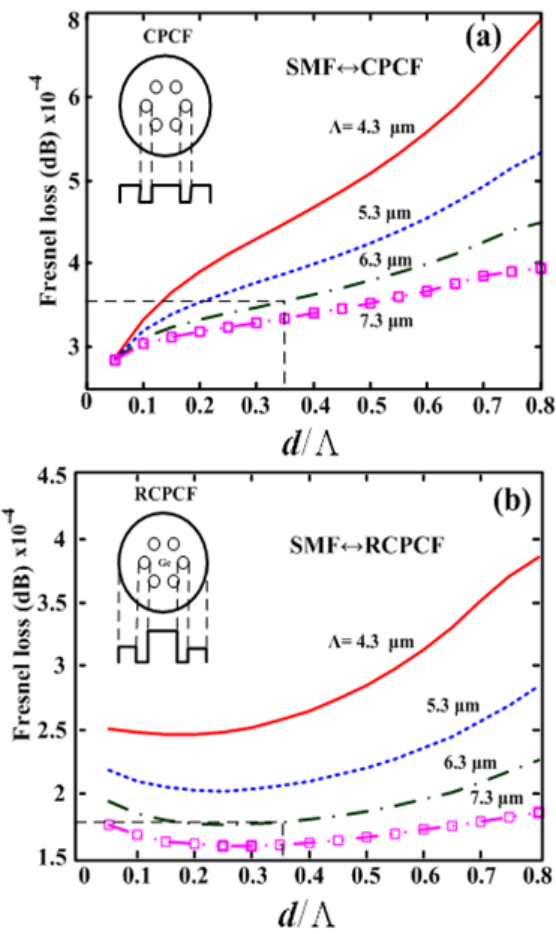

Figure 3. Calculations of Fresnel losses at perfect splice joints between SMF and (a) a CPCF and (b) an RCPCF, showing the influences of $\Lambda$ and $d / \Lambda$

As before, this condition can be extracted from Eq. (4), and Fig.4, as well, because as it is observed the value of $R$ can be smaller than the reflection coefficient of entering face of the fiber at the joint. According to the later condition, the value of $n_{2}$ is not constant rather directly depends on $\Lambda$, $d / \Lambda$, and implicitly depends on the wavelength.

In all the calculations, it is assumed the light strikes the end facets of the fibers at right angle, whereas in practice it is not so. Now, for practical case, we consider an incident angle $\theta_{1}$ for the input ray for $s$ - and $p$-polarization (See Fig. 1).

For a non-magnetic medium, the reflection coefficients of $s$-polarization $\left(R_{\mathrm{S}}\right)$ and $p$-polarization $\left(R_{\mathrm{p}}\right)$ with a non-zero incidence angle $\theta_{1}$ and refracted angle $\theta_{2}$ for $D=0$ are expressed in general form as[14]:

$$
\begin{gathered}
R_{\mathrm{S}}=\frac{\left(n_{1} \cos \theta_{1}-n_{3} \cos \theta_{2}\right)^{2}}{\left(n_{1} \cos \theta_{1}+n_{3} \cos \theta_{2}\right)^{2}} \\
R_{\mathrm{p}}=\frac{\left(n_{3} \cos \theta_{1}-n_{1} \cos \theta_{2}\right)^{2}}{\left(n_{3} \cos \theta_{1}+n_{1} \cos \theta_{2}\right)^{2}}
\end{gathered}
$$

where $n_{1}$ and $n_{3}$ are as defined in Fig. 1.

Now, by assuming $\theta_{1}=\theta_{2}$ in Eqs. (11) and (12), the effect of incident angle on $R_{\mathrm{S}}$ and $R_{\mathrm{p}}$ is determined and plotted in Fig. 5 by considering $\lambda=1.55 \mu \mathrm{m} . n_{1}=1.4701, n_{3}=1.465$, $\Lambda=6.30 \mu \mathrm{m}$, and $d / \Lambda=0.345$. It indicates that when the ray is incident at the interface between two spliced fibers with an angle of about $45 \mathrm{deg}$, the Fresnel loss will be zero. This angle is the well-known Brewster angle at which the reflectance for $p$-polarization is zero. Therefore, the validity of considering zero incident angle for small Fresnel loss may be extended to even higher incident angles. 

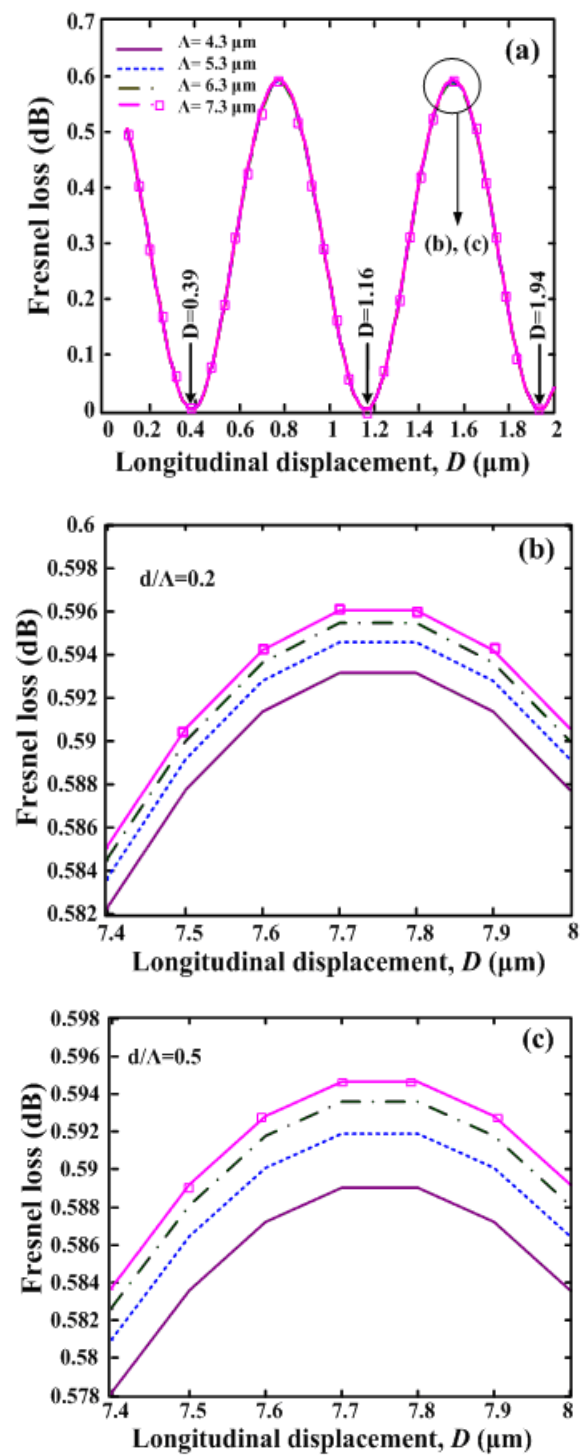

Figure 4. Variations of the Fresnel loss as a function of longitudinal displacements using IVEIM at $1.55 \mu \mathrm{m}$.

In separate plots similar to Fig. 5 for different values of $\Lambda$ and $d / \Lambda$ used for our calculations, we noted that there were almost no changes in the variations of $R_{\mathrm{S}}$ and $R_{\mathrm{p}}$. This also implies that our assumption of $\theta_{1}$ being zero is justified.

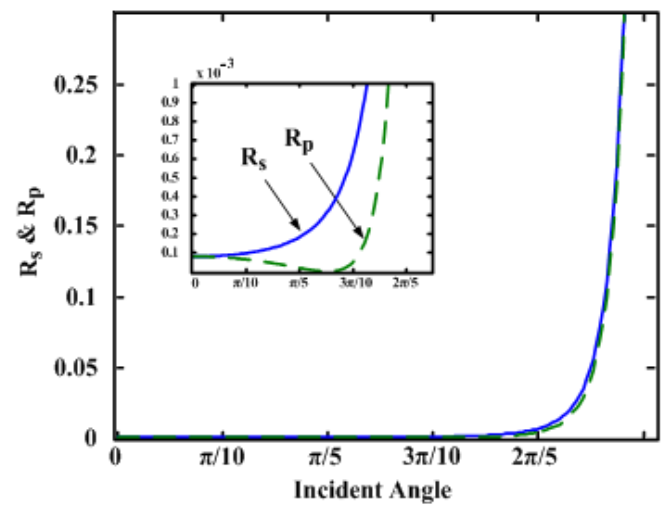

Figure 5. Reflection coefficients of $s$ - and $p$-polarization as functions of the incident angle for SMF and PCF splice joint. $\Lambda=6.30 \mu \mathrm{m}, d / \Lambda$ $=0.345 . \lambda=1.550 \mu \mathrm{m} . \quad n_{1}=1.4701, n_{3}=1.465$.
With reference to our recent reports, we note that the optimized values of $\Lambda$ and $d / \Lambda$ for the PCF-based devices lie in the single-mode region, which are the values resulting in a low Fresnel loss with a reasonable approximation based on present results in Fig. 3 [10, 17, 13].

\section{Conclusions}

We presented an analysis of Fresnel loss at splice joint of SMFs and two different types of PCFs (CPCF and RCPCF) by using improved fully vectorial effective index methods. By formulating the dependency of the Fresnel loss on the PCF parameters $\Lambda$ and $d / \Lambda$ and including the influences of effective refractive index of the fibers at the splice joint of SMFs and PCFs, the parameters ranges are determined for minimum Fresnel losses.

The analysis has shown that when the air-hole radius of spliced PCFs increases or when the RCPCF is used, there will not be a suitable method. Instead, IVEIM method will present better results.

It is shown that by increasing the effective index of the core, an increase of $\Lambda$ and a decrease of $d / \Lambda$ would reduce the Fresnel loss by more than $50 \%$. Our analysis also shows that when using RCPCF, the design and fabrication become more flexible with respect to $\Lambda$ and $d / \Lambda$. By considering a special case of $n_{1}=1.4701, n_{3}=1.465, \Lambda=6.30$ $\mu \mathrm{m}$, and $d / \Lambda=0.345$ at $\lambda=1.55 \mu \mathrm{m}$, at an incident angle of about 45 deg. the Fresnel loss will be zero. In addition, it is shown that the effect of incident angle at the splice joint on the Fresnel loss is not considerable.

To optimize the Fresnel loss at the splice joint of SMFs and PCFs, three approaches are proposed: 1) use of a material with a known refractive index at the splice joint, 2) control via longitudinal misalignment at the splice joint, and 3) control via PCF parameters $\Lambda$ and $d / \Lambda$.

With reference to our recent results[10,17, 13], we note that the optimized values of $\Lambda$ and $d / \Lambda$ for the PCF lie in the single-mode region, which are the values resulting a low Fresnel loss with a reasonable approximation based on present results.

\section{REFERENCES}

[1] F. Couny, F. Benabin, and P.S. Light, "Reduction of Fresnel Back-Reflection at Splice Interface between Hollow Core PCF and Single-Mode fiber", IEEE Photon. Technol. Lett., Vol.19, No.13, pp. 993-995, July 2007.

[2] B. Elliott and M. Gilmore, Fibre Optic Cabling, Second Edition, Newnes, Oxford, UK, 2002.

[3] S. Pradhan, A. Mazloom, J. Arbulich, and K. Srihari, Minimization of Splice Loss Between a Single Mode Fiber and an Erbium Doped Fiber", in Proc. 52nd Electron. Components and Technol. Conf., 2002, pp. 1729-1734.

[4] A. Oehler, T. Hauff, W. Heinlein, W. Stleb, J. Schulte, New 
field-matching technique for low-loss splices between conventional and dispersion-flattened single-mode fibers", in Proc. 14th Euro Conf. Opt. Commun. (ECOC 88), Vol. 1, pp. 603-606, 1988.

[5] J. T. Lizier and G. E. Town, "Splice losses in holey optical fibers", IEEE Photon. Technol. Lett., Vol. 13, No. 8, pp. 794-796, 2001.

[6] B. Bourliaguet, C. Paré, F. Émond and A. Croteau, "Microstructured fiber splicing", Opt. Exp., Vol. 11, No. 25, pp. 3412-3417, 2003.

[7] J. Lægsgaard and A. Bjarklev, "Reduction of coupling loss to photonic crystal fibers by controlled hole collapse: a numerical study", Opt. Commun., Vol. 237, Iss. 4-5, pp. 431-435, 2004.

[8] Kunimasa Saitoh, and Masanori Koshiba, "Numerical Modeling of Photonic Crystal Fibers", IEEE, J. Lightwave Technol., Vol. 23, Iss. 11, pp. 3580-3590, 2005.

[9] Ni Yi, An Liang, Xie Yuejian, Zhang Lei, Peng Jiangde, Confinement loss in solid-core photonic bandgap fibers", Opt. Commun., Vol. 235, pp. 305-310, 2004.

[10] F. E. Seraji and S. Farsinezhad, "To optimize splice joint between different PCF-based devices and SMF transmission medium", Chin. Opt. Lett. Vol. 7, No. 3, pp. 246-250, 2009.

[11] Yan-feng Li, Ching-yue Wang and Ming-lie Hu "A fully vectorial effective index method for photonic crystal fibers: application to dispersion calculation", Opt. Commun. Vol. 238, Iss. 1-3, pp. 29-33, 2004.
[12] Y. Li, Y. Yao, M. Hu, L. Chai, and Ch. Wang, "Improved fully vectorial effective index method for photonic crystal fibers: evaluation and enhancement", Appl. Opt., Vol. 47, pp. 399-406, 2008.

[13] F. E. Seraji, M. Rashidi, V. Khasheie, "Parameter analysis of a photonic crystal fiber with raised-core index profile based on effective index method", Chin. Opt. Lett., Vol. 4, No. 8, pp. 442-445, 2006

[14] J. R. Reitz, F. J. Milford, and R.W. Christy "Foundation of Elecrtomagnetic Theory", Fourth Edition, Addison-Wesley Publishing Co., N.Y., USA, 1993.

[15] J. Corbett, A. Dabirian, T. Butterley, N. A. Mortensen, and J. R. Allington-Smith,"The coupling performance of photonic crystal fibres in fibre stellar interferometry", Mon. Not. R. Astron. Soc 368, pp. 203-210, 2006.

[16] F. E. Seraji, S. Farsinezhad, L. Chehreghani, "Optimization of Long-Period Grating Inscribed in Large Mode Area Photonic Crystal Fiber for Design of Bandstop Filter", Opt. Int. J. Light Electron. Opt., 2010, doi:10.1016/j.ijleo.2010.02.004, (In press).

[17] F. E. Seraji, L. Chehreghani, S. Farsinezhad, "Design of Compact Long-Period Gratings Imprinted in Optimized Photonic Crystal Fibers", J. Appl Phys B Vol. 97, pp. 425-429, 2009.

[18] F. E. Seraji and S. Farsinezhad, "A Proposed Mechanism to Optimize Splice Joint Between PCF and SMF”, 7th Int. Conf. Opt. Comm. Net. ICOCN 08), Singapore. 